\title{
Risque de submersion et gestion participative
}

\author{
Thierry SAUZEAU ${ }^{1}$, Jacky LAUGRAUD² et Hélène PLESSIS ${ }^{3}$
}

\author{
1. Président co-fondateur de l'Université Populaire du Littoral Charentais - thierry.sauzeau@univ-poitiers.fr \\ 2. Vice-président co-fondateur de l'Université Populaire du Littoral Charentais-jacky.laugraud@wanadoo.fr \\ 3. Chargée de mission de l'Université Populaire du Littoral Charentais - chmuplc17@sfr.fr
}

\begin{abstract}
RÉSUMÉ. - La submersion de la tempête Xynthia (27-28 février 2010) a frappé les côtes du Centre-ouest français où 53 décès ont été à déplorer. Cette catastrophe, qualifiée de tempête du siècle, a beaucoup surpris les autorités. Le risque de submersion avait été oublié. Sa dernière manifestation d'ampleur comparable datait de 1957. Le développement du tourisme de masse, grâce à l'automobile, s'était effectué à l'abri de tout nouvel événement. C'est toute une politique publique qui est a inventer sur le littoral et il appartient au acteurs d'aujourd'hui d'y participer. Depuis sa création en 2011, et grâce au soutien de la Fondation de France et de la Région Poitou-Charentes, l'Université Populaire du Littoral Charentais tente de relever ce défi à l'aide de 3 leviers : la recherche, l'éducation populaire et la participation. Il a d'abord fallu recueillir les résultats de la recherche mais aussi animer des travaux pluridisciplinaires de haut niveau. Ensuite la présentation de ces travaux, au plus près du terrain, a permis de recueillir les réactions du public. Un nouveau corpus documentaire, fait de résultats scientifiques et d'expériences individuelles, a finalement été produit afin d'éclairer les politiques publiques, au plus près du citoyen.
\end{abstract}

Mots-clés : submersion, risque, gestion, participation.

\section{Risk of submersion and participative management}

\begin{abstract}
Flooded Xynthia (27-28 February 2010) hit the French center-west coast where 53 deaths were reported. Due to the event, our societies have replayed a drama in several acts. This disaster, described as the storm of the century, much surprised the authorities. The risk of flooding had been forgotten. His last event of comparable magnitude dated from 1957. The development of mass tourism through the automobile, was performed at the shelter of any new event. This is a public policy that has to be invented on the litoral, and it is up to current residents to participate. Since its founding in 2011, and with the support of the Fondation de France and Poitou-Charentes Region, People's University of the Littoral Charentais trying to meet this challenge with three levers: research, public education and participation. It was first necessary to collect research results but also to facilitate a multidisciplinary work of high level. Then the presentation of the work, as close to the territory, raised public reaction. A new documentary corpus, made of scientific results and of individual experiences, has finally been produced to enlight public policy, closer to the citizen.
\end{abstract}

Key-words: submersion, risk management, participation.

\section{INTRODUCTION}

La tempête Xynthia (27-28 février 2010) a traumatisé les populations du Centre-ouest français. Outre les 53 décès à déplorer, les dégâts ont été considérables : maisons détruites, réseaux endommagés, terres agricoles noyées. L'emballement médiatique, qui suit chaque catastrophe, a fait de Xynthia un signe annonciateur des changements climatiques à venir alors même que ce fait est loin d'être scientifiquement établi [Chaumillon et al., 2014]. Pour réagir à un événement, l'Etat a mis en œuvre une politique d'abandon à la mer conçue pour faire face aux changements graduels [Goeldner, 2013]. Malheureusement, effectué trop rapidement et avec des moyens inadaptés, ce travail a été dénoncé. Les spécificités du littoral charentais expliquent l'ampleur des réactions. Ville universitaire, La Rochelle, s'est retrouvée au cœur de la catastrophe. Ses chercheurs, familiers des problématiques littorales et forts d'une expertise reconnue, ont fait connaître la complexité du phénomène et dialogué avec les populations des zones submergées. Profondément renouvelées, et dominées par des retraités aisés et de jeunes actifs du secteur tertiaire, ces communautés du début du $\mathrm{XXI}^{\mathrm{e}}$ siècle se sont révélées orphelines d'une histoire collective et vierges de toute conscience du risque mais leurs habitants partagent un bon niveau culturel, servi par la révolution du numérique et de l'information. C'est sur un tel terrain que plusieurs initiatives se sont développées.

Spécialisés dans l'histoire des paysages sur le temps long, des chercheurs de l'université de Poitiers, ont produit un modèle de contre expertise, début avril 2010, résultat d'une expérience de science participative appliquée au village de La Perrotine (île d'Oléron) et à sa zone noire. L'enquête s'est organisée autour de l'analyse historique des archives et des cartes anciennes. Elle s'est ensuite nourrie du recueil des témoignages des victimes et des souvenirs des habitants enracinés. Dans les semaines qui ont suivi la catastrophe, ce modèle d'étude a été intégré à un ouvrage collectif, paru sous la caution scientifique d'Emmanuel Garnier [Garnier et Surville, 2010]. Cet ouvrage était la synthèse d'un rapport 
adressé au législateur [Garnier, 2010], où la banalité des événements extrêmes météo marins dans l'histoire était renvoyée à la capacité des riverains de les assumer.

Il y avait là de quoi nourrir et construire une action publique alternative, au plus près du terrain. Le Conseil Régional de Poitou-Charentes a financé des bourses de Master afin de lancer des recherches, sur le modèle de La Perrotine, dans six autres zones noires. Port-des-Barques a fait partie des 6 villages inclus dans ce programme. Fort de la connaissance intime de son territoire, son maire Jacky Laugraud s'est appuyé sur les résultats pour demander, non pas la suppression, mais l'extension de « sa » zone de solidarité. Il s'agissait d'un groupe de lotissements récents situés dans une zone humide présente - et vide - sur tous les documents depuis le XVII siècle, le Maréchat [Sauzeau, 2014.a]. C'est de la rencontre entre cet élu et les chercheurs, dans un contexte de vulgarisation scientifique vers le grand public que sont nés l'Université Populaire du Littoral Charentais (UPLC) et son projet.

Les statuts de l'association proclament qu'elle rassemble des chercheurs, des élus, des associations, des entreprises et des citoyens intéressés au devenir du littoral charentais, pour préparer l'avenir en partageant les savoirs. La reconstitution de la trajectoire individuelle de chaque territoire face à la mer, enrichie par les multiples dimensions des patrimoines paysager, bâti et immatériel (bonnes pratiques) est la base conceptuelle du programme baptisé "Vision collective et gestion participative du risque ", honoré d'un financement pluriannuel par la Fondation de France (2013-2015) et soutenu par la Région Poitou-Charentes.

Comment éclairer la prise de décision sur le littoral, en intégrant les visions collectives des habitants et les acquis des recherches les plus actuelles ? C'est le cœur du sujet traité dans cet article qui expose la méthodologie développée à l'UPLC. On présentera d'abord les modalités de collecte et de diffusion de l'information scientifique relative au Centre-ouest atlantique. Une nécessaire validation des savoirs, parmi la profusion des ressources numériques accessibles, constitue un préalable. Il s'agit aussi d'assurer la publicité de connaissances acquises sur fonds publics mais restant top souvent confidentielles. Dans un second temps, c'est la question du transfert vers les acteurs du littoral qui doit être traitée. La démarche est celle de l'éducation populaire. Elle s'attache à varier les échelles du discours : du temps long à l'événement, du global au local. Enfin, la présentation des résultats permet d'organiser une collecte des réactions du grand public. La démarche est itérative. Cette collecte constitue à la fois un instrument de mesure de l'appropriation de la question du risque par les riverains mais elle est riche de nouvelles approches, notamment basées sur la mémoire, à développer désormais.

\section{L'APPROPRIATION DE SAVOIRS PLURIDISCIPLINAIRES.}

Le premier temps de notre action a été marqué par l'appropriation d'un savoir pluridisciplinaire de haut niveau. Ces connaissances sont collectées à trois niveaux. Elles sont pour partie produites au sein même de l'association par le biais de stages d'étudiants de Master. Elles sont aussi collectées par une veille scientifique effectuée tant au sein du réseau d'universités partenaires (La Rochelle, Nantes, Poitiers), que dans les territoires (rapports de bureaux d'études) ou auprès du Ministère de l'Ecologie, du Développement Durable et de l'Energie (MEDDE). Un premier résultat a pris la forme d'une publication mise en chantier, et menée à bien, afin de valoriser le travail de reconstitution des trajectoires de territoires [Péret et Sauzeau, 2014].

\section{II.1. Du travail de veille documentaire à la base de ressources en ligne.}

C'est par l'intermédiaire du dispositif « emploi-tremplin » de la Région et grâce aux crédits pluriannuels octroyés par la Fondation de France que les premières bases du programme ont pu être jetées. Notre chargée de mission, Hélène Plessis, a été engagée sur la durée du contrat (2013-2015). Un poste de chargée de valorisation des ressources documentaires a été créé pour une durée de six mois. Il s'agissait d'accompagner le travail des bénévoles du conseil d'administration dans deux domaines.

On a d'abord initié un travail de collecte de l'information scientifique, technique et diffusée par voie de presse. Dans ce cadre, des abonnements au quotidien Sud-Ouest et à 3 hebdomadaires du littoral ont été contractés. Par ailleurs, un effort financier a été consenti pour pouvoir être présent sur des manifestations scientifiques (colloques, journées d'études), en co-financer certaines (organisées par des associations ou des institutions adhérentes) mais aussi communiquer nos propres résultats. Depuis sa prise de fonction en 2013 en tant que chargée de mission, Hélène Plessis a ainsi participé à 13 colloques. Les thèmes abordés au cours de ces sessions étaient très variés. Ils ont permis à l'association de former ses cadres bénévoles et sa salariée à la prise en compte globale des problématiques du risque littoral. La plupart des sessions était consacrée à ce thème du risque maritime et littoral (submersion, érosion) : «Submersions marines : Quelles stratégies envisagées ? Quels ouvrages concrets ? ", "Quand gestion hydraulique rime avec continuité écologique dans les zones humides littorales », « Populations et activités sur le littoral atlantique : Enjeux fonciers - Quelle gouvernance avec quels outils ? ", les Assises Nationales des Risques Naturels, "Les risques littoraux et maritimes », « Réduire les risques littoraux et s'adapter au changement climatique », « Submersions marines : Passé, Présent, Futur », « Hausse du niveau de la mer et risques côtiers », " Connaissance et compréhension des risques côtiers : Aléas, Enjeux, Représentations, Gestion ». D'autres sessions ont été suivies afin de s'approprier les connaissances les plus actuelles en matière d'environnements côtiers : "Partager la mer : pêche, aquaculture et autres usages », «Qualité des eaux littorales : suivi et enjeux », « La recherche en Histoire maritime : enjeux, objets et méthodes », Ateliers du Conservatoire du littoral : «Retours sur investissement : La contribution des espaces naturels littoraux à l'économie des territoires ».

Le fil rouge de ces réunions scientifiques est le constat d'une évolution des usages au gré des sociétés qui se sont installées à proximité du littoral et dont les attentes ont eu pour conséquence de modifier les pratiques et les paysages. Par voie de conséquence, le littoral connaît aujourd'hui des tensions, des conflits ou des dégradations liés notamment au développement du tourisme et des activités récréatives aux dépens de certaines activités primaires (conchyliculture, pâturage). La question de l'adaptation des populations face au changement climatique se pose du fait de l'évolution des modes de vie sur le littoral qui ont conduit à davantage exposer les populations et les biens aux risques littoraux (érosion et submersion) et donc à les rendre plus vulnérables face à ces phénomènes. Face à ces questions, il existe des débats. Convient-il de "protéger plus » (construire plus 
d'ouvrages de défense contre la mer) ? Au contraire, ne vaudrait-il pas mieux « laisser la mer reprendre ses droits » en localisant les biens et les activités dans les zones rétrolittorales, moins exposées aux aléas ? Une autre option, connue sous le nom de " mitigation ", consisterait à organiser un recul graduel des enjeux humains, en faisant évoluer l'existant et en stoppant toute nouvelle densification des enjeux (bâti, réseaux, activités.)

Dans un second temps, notre objectif de diffusion de l'information a commandé la mise en œuvre d'une base de ressources électroniques en ligne. Pour mener à bien cette tâche, nous avons fait confiance à la titulaire d'un double diplôme franco-québécois dans le domaine de la documentation et de l'archivistique. Sous contrat pour 6 mois, Amélie Giret a dressé le cahier des charges, lancé un appel d'offre et c'est finalement une solution en open source qui a été privilégiée. A l'heure actuelle la base est accessible depuis la page d'accueil du site internet (www.uplc17.org) et met un millier de notices à la disposition du grand public, grâce à un moteur de recherche avancée. Si les articles sont majoritairement issus des revues de presse (notamment la rétrospective complète du « dossier » Xynthia depuis 2010) le défi consiste à présent à mettre en ligne l'importante moisson d'articles scientifiques et de documentation technique.

\section{II.2. De l'hébergement de stages d'étudiants à la publication d'une synthèse.}

Le projet honoré par la Fondation de France intégrait également l'hébergement et la direction de stagiaires dans le cadre associatif. Trois stagiaires de Master en géoscience et en géographie, inscrits à l'université de La Rochelle ont été engagés en 2013 pour une durée cumulée de 14 mois. Trois nouveaux stagiaires ont été engagés en 2014, un géographe rochelais et deux poitevines (histoire et géographie) pour une nouvelle durée cumulée de 14 mois.

Le recensement et la caractérisation des événements extrêmes survenus en Centre-ouest depuis le $\mathrm{XVI}^{\mathrm{e}}$ siècle ont occupé 2 de ces stages, sur 12 mois cumulés tandis que 16 autres mois ont été consacrés à la reconstitution de trajectoires de territoires face à la mer. Ce travail de production de recherche est venu conforter celui initié par les 6 bourses de Master financées en 2010-2011 par la Région. Au printemps 2014, l'ouvrage intitulé Xynthia ou la mémoire réveillée est paru chez Geste éditions avec le soutien de la Région et sous l'égide de l'UPLC. Ce sont les histoires collectives, depuis le $\mathrm{XVI}^{\mathrm{e}}-\mathrm{XVII}{ }^{\mathrm{e}}$ siècles jusqu'à nos jours, de 14 sites et villages ruraux du Centre-ouest français qui ont été publiées. Dans son chantier de recherche participative, l'UPLC dispose désormais d'une série d'études de cas (et aussi de productions inédites) propres à amorcer un dialogue avec les acteurs de terrain. En effet, les résultats de ses propres recherches, accompagnés par la synthèse des travaux pluridisciplinaires récoltés, ouvrent la voie à des restitutions dans les villages profondément transformés par la révolution balnéaire.

\section{ANIMER LA RÉFLEXION SUR LES TERRITOIRES DU RISQUE}

La seconde phase du processus s'appuie sur la constitution d'un corpus d'informations souvent inédites et systématiquement dispersées. Elle s'attache à la construction d'une vision collective basée sur l'histoire et permet d'appuyer les travaux menés dans les territoires dans le domaine de la prospective. La présentation de synthèses issues de la veille documentaire réglementaire, technique et scientifique rejoint en effet la présentation des trajectoires des villages littoraux du Centre-ouest. La phase en cours passe par une série de manifestations destinées à faire partager l'état des connaissances récoltées selon trois modalités. A l'égard de la communauté scientifique, l'UPLC s'est appliquée à passer du statut d'auditeur passif à celui de partenaire actif. Nous contribuons au montage de manifestations et figurons même comme partenaire dans des programmes de recherche. Vis-à-vis du grand public, deux types d'animations scientifiques sont initiées. Depuis l'automne 2012, l'UPLC tient ses «Rencontres littorales » sur des thèmes d'intérêt général et depuis le printemps 2014 une formule, adaptée à des quartiers ou des villages, se développe sous l'appellation "Rendez-vous du littoral », avec l'appui d'associations locales adhérentes et partenaires.

\section{III.1. Participations et partenariats scientifiques (colloques, thèses, collaborations)}

Quoique portée sur les fonts baptismaux à la faveur d'une crise et bien que ses parrains aient été entraînés dans une controverse aiguë, l'UPLC a acquis une réputation de sérieux, d'indépendance et de qualité pour les travaux qu'elle initie ou qu'elle favorise. L'intérêt suscité dans la communauté scientifique en témoigne.

L'association a été associée au montage de manifestations scientifiques. Elle a figuré au rang des partenaires du colloque «De la mer au désert : histoire des environnements littoraux » (2013, CRiHAM, Limoges/Poitiers) et du colloque réuni autour de la question de la réduction des risques littoraux et de l'adaptation face au changement climatique (LIENSs, La Rochelle, 2014.) Le programme pluridisciplinaire « dynamique des changements d'usages conchylicoles sur le fonctionnement des écosystèmes littoraux » (DYCOFEL 2015-2017), honoré d'un financement pluriannuel par la Fondation de France, intègre l'UPLC en amont (collecte d'information et de données) comme en aval (restitutions et partage des résultats.) Il associe des biologistes, des économistes, des juristes, des géographes et des historiens.

Une série de publications scientifiques fait rayonner la recherche menée en interne (stages) ou au sein de programmes pluridisciplinaires. Un papier paru en 2012 dans la revue géographique Norois [Acerra et Sauzeau, 2012], a été consacré aux questions de gouvernance des zones littorales sur le temps long (XVI ${ }^{\mathrm{e}}-\mathrm{XXI}^{\mathrm{e}}$ siècles). On a montré que la valeur de l'expérience de terrain n'échappait pas à des pouvoirs pourtant bien éloignés de notre démocratie contemporaine : la monarchie absolue ou le second Empire ont été plus attentifs que nous ne le sommes à la parole des experts du quotidien. Un autre article, suite à une intervention dans un colloque d'histoire de l'environnement, a permis de développer l'intérêt de la mémoire et de l'histoire des quartiers et villages. Une grammaire de la dynamique des paysages littoraux a été esquissée [Sauzeau, 2014.b]. Elle offre une aide à la décision face aux changements. En 2014, un article est paru avec le soutien de l'UPLC, dans la revue internationale Global planetary and changes qui a validé l'usage avancé des réanalyses atmosphériques pour reconstituer des tempêtes du passé, vers la production d'une classification des tempêtes dans le Centre-ouest français.

Le sérieux des études et des analyses conduites au sein de l'association nous permet aussi d'être considéré comme un appui légitime pour des thèses en cours, et ce dans plusieurs disciplines. Directement issue de son stage de Master 1, lié au programme «Xynthia » de la Région Poitou-Charentes, 
la thèse de géohistoire que Pierre Caillosse consacre à la pointe de Soulac/Le Verdon, du XIII ${ }^{\mathrm{e}}$ au $\mathrm{XX}^{\mathrm{e}}$ siècles s'inscrit dans ce cadre. Elle sera soutenue à l'automne 2015 au laboratoire LIENSs de La Rochelle. Membre de l'UPLC depuis 2012, le laboratoire nantais Géolittomer abrite quant à lui deux recherches doctorales auxquelles 1'UPLC est associée. Il s'agit d'abord des travaux de géographie du risque qu'Axel Creach consacre à la vulnérabilité du littoral vendéen. Il en va de même du travail d'histoire de l'environnement qu'Emmanuelle Athimon consacre aux tempêtes XIII ${ }^{\mathrm{e}} \mathrm{XVII}{ }^{\mathrm{e}}$ siècles dans le cadre du royaume de France. Enfin, après avoir enchaîné deux stages de Master 1 et 2 à l'UPLC et dans une association partenaire, Marie Cloutour est engagée dans une thèse d'histoire consacrée à la trajectoire de la Saintonge maritime face à la mer au $\mathrm{XVII}^{\mathrm{e}}$ siècle, recherche conduite au sein du CRiHAM, université de Poitiers.

Les connaissances acquises au sein même de l'UPLC sont donc mobilisées, au même titre que la capacité de l'association à fournir une assistance aux doctorants et aux chercheurs confirmés. Le bottom up voit l'expertise de terrain se mettre au service de la production scientifique. Le top down voit la recherche scientifique venir nourrir la réflexion des citoyens, des habitants et des usagers du littoral grâce à une très active politique de vulgarisation.

\section{III.2. Les « Rencontres littorales » : partager les savoirs pour préparer l'avenir.}

C'est dans ce souci de mettre les adhérents et le grand public au fait des dernières avancées scientifiques et des stratégies actuelles sur le littoral que les Rencontres sont organisées chaque année courant novembre. Nous tenons compte du calendrier des marées, de manière à donner aux conchyliculteurs la possibilité de participer. Avec l'appui de partenaires historiques tels que le Centre international de la mer, la Chambre de commerce et d'industrie du pays rochefortais, le Forum des marais atlantiques, la MAAF ou bien 1'Etablissement Public Territorial de Bassin du fleuve Charente, des ateliers ouverts à tous sont organisés. L'objectif est de favoriser les échanges entre les intervenants et le public constitué de citoyens, d'élus, d'étudiants et de représentants de diverses structures (Communautés de communes et d'agglomération, associations, universités, etc). Après une première édition sur le thème "Vivre le littoral avec la mer : des risques naturels ... et après ?" (2012), l'UPLC a organisé ses rencontres suivantes sur "Les métiers du littoral : Aujourd'hui et demain ? » (2013) avant de sonder les questions de gouvernance : "Qui décide sur le littoral ? » (2014). Force est de constater que ces manifestations mobilisent l'ensemble des acteurs du littoral puisque, chaque année, on dénombre 100 à 120 participants et un nombre encore plus grand de curieux téléchargent les comptes-rendus mis en ligne sur le site de l'association.

\section{III.3. Les « Rendez-vous du littoral » : le récit collectif au secours de la vision du risque.}

La connaissance, qu'elle soit engrangée à la faveur de nos participations diverses, ou bien produite dans le cadre de nos propres activités, a vocation à être partagée. C'est l'objet des Rencontres annuelles mais aussi, de manière plus ciblée, la raison d'être des Rendez-vous du littoral. Les élus, les agents de l'Etat ou des collectivités, les habitants de plus ou moins fraîche date constituent le public désigné de ces restitutions.
Dans ce cadre particulier, nous nous emparons de problématiques globales afin de les décliner à l'échelle locale. Autant que possible, nous tentons de rompre avec les échelles inadaptées et nous articulons les modélisations du risque avec l'histoire du village-hôte face à la mer. Il s'agit de territorialiser les enjeux afin de rapprocher la présentation des habitants. Le propos s'adapte au territoire vécu de manière à libérer la parole. Chaque soirée est conçue sur le même modèle, en trois temps. On commence par exposer la richesse de la documentation disponible pour traiter des événements extrêmes et des changements graduels, sur le temps long. On présente les prospectives les plus actuelles concernant les environnements littoraux d'entre Loire et Gironde. Enfin, au gré des localités d'accueil de ces manifestations, tenues à l'initiative conjointe de l'UPLC et d'une association partenaire, on présente des diagnostics sur mesure et aussi actualisés que possible. Les 3 temps de la présentation sont brefs et l'échange est favorisé. Venu s'informer sur la vulnérabilité de son territoire, le public de ces «Rendez-vous $d u$ littoral » est mis en face de son histoire collective face à la mer et il est amené à l'enrichir de ses propres souvenirs, connaissances ou traditions.

A partir de l'automne 2014, ce travail a été initié sur les sites de Saint-Georges de Didonne (rive nord de la Gironde) et Dolus (île d'Oléron). Nous le poursuivons durant ce printemps 2015 à Port-Neuf (agglomération de La Rochelle), Saint-Trojan (Oléron), Port-des-Barques et Barbâtre (île de Noirmoutier). C'est l'occasion de recueillir des réactions, d'enregistrer des récits et des souvenirs qui viennent enrichir les connaissances disponibles sur la trajectoire des territoires visités. Cela permet aussi d'engager la discussion entre les décideurs et la population, voire de lancer des initiatives d'un nouveau type, entre vision collective et gestion participative du risque littoral.

\section{FAVORISER UNE PRISE DE DÉCISION ÉCLAIRÉE SUR LE LITTORAL}

La troisième phase du travail conduit à l'UPLC touche à la gestion participative et suit une série de pistes très prometteuses. Il s'agit d'organiser le transfert des connaissances issues de la recherche, enrichies par les échanges avec les acteurs locaux, afin de lancer des initiatives concrètes et utiles à la gestion du risque. Les actions de formation offrent une première modalité de transfert auprès de publics demandeurs (élus, agents des collectivités locales). A une autre échelle, l'insertion professionnelle des stagiaires accueillis à l'UPLC entretient et assure l'application de terrain d'une recherche pensée et coordonnée au sein de l'association (base de données historiques des submersions marines, trajectoires de territoires ou de segments littoraux sensibles). Enfin, et surtout, la valorisation du legs de bonnes pratiques, dont témoignent encore certains habitants enracinés, offre de nouvelles pistes de valorisation. Les paroles tirées de l'expérience, en dialogue avec les acquis de la recherche scientifique, semblent appelées à jouer un rôle essentiel dans la médiation face aux dynamiques de changement comme dans la prévention des risques.

\section{IV.1. Former les citoyens, les acteurs et contribuer à la prise de décision.}

Depuis 2012, nos actions de collecte, de recherche et de transfert des connaissances vers le terrain, sous la forme de « Rendez-vous du littoral » ou de « Rencontres littorales » 
initient une double appropriation de la connaissance. Parmi les résultats concrets et opérationnels, il y a le transfert de ces connaissances forgées dans le dialogue entre la recherche et la parole citoyenne, en direction des cercles de décision.

Investi à titre professionnel comme Chargé de mission Littoral pour la Région Poitou-Charentes Jacky Laugraud, met à profit ses interventions pour maintenir le cap de l'action de l'UPLC, au carrefour des préoccupations de ses fondateurs, au bénéfice de ses adhérents. Au sein de l'atelier national " paysages et aménagements post inondations » et de l'atelier « résilience des territoires » du MEDDE, plusieurs notions travaillées dans le cadre associatif sont défendues. La prise en compte des zones humides comme zones d'expansion de crues et de submersions constitue un axe fort. Les communications réalisées dans ce cadre sont vulgarisées vers les publics non élus ou techniciens : les apports de ces ateliers sont systématiquement enregistrés dans la base de ressources en ligne de l'UPLC. Par ailleurs, un lien permanent est entretenu avec des institutions et acteurs d'échelle nationale (Commission Mixte Inondations, Groupement d'Intérêt Public du Littoral Aquitain, représentants français au GIEC, CNFPT). Tout est mis en œuvre pour garder au citoyen sa place dans le débat public. A Port-des-Barques, l'action municipale du mandat 2008-2014 continue d'être relayée au présent par l'action associative (écomusée ; association « Sport, Culture et Découvertes »). Le lien avec une population directement impactée par les aléas passés est maintenu. Le débat autour des solutions de prévention à mettre en oeuvre avec la population est entretenu.

L'UPLC participe par ailleurs à l'animation de sessions de formation (Lycée de la Mer de Bourcefranc, Université de La Rochelle, CNFPT). A travers des études de cas et des actions concrètes, elle permet aux publics cibles d'envisager les problèmes d'aménagement post-crises et la prospective dans le sens de l'aménagement durable des territoires littoraux. Ce type d'action de formation / information est décliné en direction du grand public mais aussi des élus. Au-delà du terrain portbarquais, qui constitue un solide laboratoire, la communauté de communes de l'île d'Oléron est un partenaire très impliqué. L'UPLC a pleinement pris sa place dans le montage scientifique de l'exposition itinérante qui circule actuellement dans les 8 communes du territoire sous le titre "Littoral [in]domptable ». Cette action est partie intégrante du Programme d'Action de Prévention des Inondations (PAPI) de l'île d'Oléron. Elle trouve sa place dans l'axe 1 «Amélioration de la connaissance et de la conscience $d u$ risque ». Jacky Laugraud a également impliqué 1'association à la réflexion autour du PAPi de l'estuaire de la Charente, dans le cadre de son mandat de vice-président de la Communauté d'Agglomération du Pays Rochefortais. A Oléron, sur une vingtaine de panneaux, 8 ont été directement nourris par la vision collective du territoire littoral, construite à partir de nos travaux. Par ailleurs, et durant la phase de montage de cette exposition, deux journées de formation ont été animées en septembre 2014, à destination des nouveaux élus municipaux. La première journée s'attachait à présenter les enjeux du littoral insulaire sous l'angle des processus naturels qui l'affectent. La seconde a été consacrée aux interactions humaines, volontaires ou involontaires, et à leurs conséquences sur l'interface terre-mer.

Les processus de décision et de gouvernance du littoral face au risque maritime nécessitent des études et des recherches avancées Elles sont le domaine d'officines et de laboratoires universitaires spécialisés. A son actif, l'UPLC a néanmoins plusieurs actions de formation et de médiation qui trouvent leur place au sein des dispositifs de planification. Notre action contribue à pallier l'absence d'un chaînon manquant afin de mieux faire partager les décisions issues d'un processus de partage, de contribution voire de co-construction, au gré des territoires.

\section{IV.2. Vers une recherche appliquée : l'insertion professionnelle de nos stagiaires.}

Deux stagiaires accueillis à l'UPLC ont eu la chance de voir des employeurs s'intéresser à leurs travaux conduits dans le cadre associatif et valorisés dans le champ scientifique. Nathalie Giloy a ainsi consacré ses 6 mois de stage à la caractérisation des événements météo-marins extrêmes à partir de données historiques, d'observations météorologiques et de réanalyses atmosphériques. Son travail portait sur la mise en place d'une base de données des événements météo-marins extrêmes ayant touché le Centre-ouest (tempêtes, submersions, tempêtes et submersions) depuis les années 1500 jusqu'à nos jours. Un volet de sa mission consistait à caractériser ces événements à l'aide de différents types de données (littéraires, instrumentales), afin de pouvoir les comparer à la tempête Xynthia en 2010. Ses conclusions ont nourri la publication d'un article en anglais [Breilh et al., 2014]. Sur la base de ce travail, elle a été recrutée en tant que prestataire pour l'IRSN (Institut de Radioprotection et de Sureté Nucléaire) au sein de l'équipe BEHRIG (Bureau d'études Bureau d'expertise hydrogéologique, risques d'inondation et géotechnique). Ses deux axes de travail sont l'étude des surcotes issues de tempêtes et ayant généré des submersions mais aussi l'évolution à long terme du niveau marin. Cette étude doit être réalisée sur la côte Manche Mer du Nord ainsi que la côte Atlantique et plus précisément dans les zones comptant des installations nucléaires au bord de mer (Gravelines 59, Penly et Paluel 76, Flamanville 50 et le Blayais 33.) La tâche de recensement des événements de tempêtes ou des submersions passées inclut bien évidemment l'emploi de la base de données établie au sein de l'UPLC et l'association pourra être sollicitée comme partenaire régional pour le site du Blayais.

Quant à Charly Point, ce sont ses compétences pluridisciplinaires en termes de traitement des données d'archive, de confection de cartes et de supports graphiques qui ont convaincu la communauté de communes de l'île d'Oléron. Son contrat actuel est destiné à la confection d'un atlas historique et dynamique de plusieurs points sensibles de l'île. L'objectif est double. Il s'agit de dresser la chronologie la plus fine possible des travaux nécessités par les ouvrages actuels, en déterminant, chaque fois que les archives le permettent, le statut des aménagements en question. A la faveur de ce travail, la communauté de communes entend anticiper la mise en œuvre de la compétence Gestion des Milieux Aquatiques et Prévention des Inondations (GEMAPi) qui devrait lui être dévolue dès janvier 2016. Dans un second temps, l'atlas historique et dynamique de micro bassins de risque (baie de La Perroche coté océan, anse de La Malconche côté continent) se consacre à la localisation et à la description précise de phénomènes ayant affecté les littoraux retenus. Il doit permettre de communiquer autour des contraintes d'urbanisme et des prospectives en termes de défense (ou d'abandon) de portions du littoral.

Ces deux contrats décrochés par nos stagiaires contribuent à construire au plus près du terrain, les solutions personnalisées à des problématiques actuelles, souvent héritées de décisions peu éclairées. La méthodologie suivie passe par l'appropriation et la vulgarisation des connaissances les plus avancées mais aussi des prospectives les plus actuelles. La prise en compte de l'histoire des territoires sur le temps 
long ancre ces analyses dans la trajectoire spécifique de la communauté ou du site. Nouvelles tâches ou nouveaux métiers, ces contrats replacent directement les problématiques actuelles (les études et la prospective) dans des cadres de réflexion à la fois plus larges (l'histoire) mais aussi plus proches des citoyens (le territoire vécu).

\section{IV.3. Collecter la mémoire du " vivre en paix » avec le littoral : un enjeu prioritaire.}

En zone littorale, la prise de décision éclairée tend de plus en plus à associer les études avancées en termes de prospective (géologie, océanographie, climatologie, météorologie, géographie) et les recherches en histoire globale appliquée à une échelle locale (géohistoire, histoire sociale, économique, politique, environnementale, ...). L'UPLC a pris sa part du processus de construction d'une vision collective propre à permettre une gestion participative du risque. Les travaux menés ont finalement souligné la valeur de la parole des anciens, non pas tant comme source de sagesse indiscutable, mais bien comme levier de médiation vers une décision publique éclairée.

Chaque acteur du littoral entretient avec son espace de vie ou de travail un triple rapport. L'affectivité ou l'émotion sont devenus le rapport dominant depuis l'avènement de la société des loisirs. La côte porte en elle des valeurs d'esthétisme, de bien être et de souvenirs partagés. Ce rapport là s'accomplit dans la mise en valeur du foncier, par et pour les nouveaux habitants. La puissance des filières du tourisme, du commerce, du bâtiment ou des travaux publics s'oppose à tout discours alternatif. Le risque est de ceux là. A chaque scrutin, la question du risque littoral (érosion, submersion, qualité de l'eau) fait courir un risque politique aux élus. Même avec la meilleure volonté, ils ne peuvent qu'y être sensibles. La seconde valeur portée sur le littoral est diamétralement opposée. Elle émane de la communauté scientifique. La biodiversité, les changements climatiques, les modes de développement et leurs conséquences sont évalués par des universités du littoral, des agences et institutions officielles. Elles produisent d'importantes contributions qui peuvent éclairer la prise de décision mais dont l'appropriation « grand public » reste limitée. L'émotion et la science ne se rencontrent pas facilement. C'est autour d'un $3^{\text {e }}$ et dernier type de rapport à la mer et au littoral que peut se nouer le dialogue. La clé réside dans l'expérience, le rapport à la mer et au littoral que nourrissent les professionnels.

Parmi eux, celles et ceux qui ont vécu les profondes mutations des années 1960-2015 peuvent jouer le rôle de passeurs entre science et émotion. L'exemple vient, dans un tout autre domaine, des associations de déportés et de résistants. Les survivants continuent inlassablement d'intervenir dans les lycées, quand bien même notre émotion face à la déportation reste intacte et alors même que le travail scientifique de diffusion de la connaissance n'a jamais cessé. De même, dans chacune de nos réunions publiques, les valeurs conjointes de la mémoire, de l'expérience vécue, des usages (pratiques professionnelles ou de loisirs, éléments de langage, savoir-faire, ...) et d'une connaissance vernaculaire des risques se sont imposées. L'objectif que nous souhaitons poursuivre à présent consisterait à collecter, pour la préserver, cette mémoire afin d'enrichir l'histoire collective des villages et quartiers littoraux. Pour remplir cette mission nous nous appuierons sur le réseau d'acteurs créé depuis plus de 3 ans et nous effectuerons un travail particulier sur les récits liés à la mémoire du risque. Les habitants ayant vécus la tempête Xynthia en février 2010 constituent un premier cercle de témoins autour duquel nous avons entamé le recensement d'interviews détenues par des musées, des associations, des médias. Actuellement le conseil scientifique de l'UPLC travaille à l'élaboration d'un questionnaire adapté en fonction de l'interlocuteur (catégorie professionnelle, usagé historique, victime de submersion marine, ...). Une autre piste de collecte pourrait être envisagée en impliquant une radio locale. Les témoignages seront collectés sous forme audio ou vidéo. Ils seront retranscrits afin de faciliter la recherche indexée et seront disponibles sur nos espaces web (site internet et base documentaire). Quant à la valorisation elle devrait s'appuyer sur des organismes relais tels que les musées (Oléron, Saint-Martin-de-Ré, Muséum d'histoire naturelle de La Rochelle ...) ou les pôles natures (Loix-en-Ré, Port des Barques ...) relevant tous à divers titres de collectivités assumant des compétences dans le domaine du risque et de sa connaissance. Nous pourrions aussi mobiliser la Ligue de Protection des Oiseaux, les réseaux des Pays d'Art et d'Histoire mais aussi faciliter l'accès pour les chercheurs (Universités, Archives Départementales ...) en assurant la mise à disposition la plus large.

\section{CONCLUSION}

A l'heure actuelle, l'occupation des littoraux connaît une densification accélérée mais ce phénomène a débuté, avec des agendas variables selon les villages et quartiers concernés, entre le XIX ${ }^{\mathrm{e}}$ siècle et les années 1960. Les mutations récentes ont fait vaciller l'état d'équilibre entre la mer et l'homme. Les aménagements et le développement économique se sont emparés de zones longtemps laissées vierges de tout enjeu, compte tenu de l'expérience du risque subie par des générations de riverains. Comme l'a souligné la submersion de Xynthia, des milliers d'habitants sont aujourd'hui vulnérables face à la submersion, sans que l'on soit capable de dire avec quelle fréquence ce risque s'exprimera demain. Les changements climatiques sont l'autre héritage des dernières décennies : l'homme modifie le climat, le réchauffement est sans équivoque. Le niveau des mers monte au rythme actuel de $3 \mathrm{~mm}$. par an, les climats de houle se renforcent dans l'Atlantique nord, l'érosion des côtes connaît une accélération. La gestion de tous ces risques littoraux pose un vrai problème de gouvernance. Comment faire partager le constat d'un risque grandissant et recueillir un accord sur les moyens d'y faire face ? La voie tracée par l'UPLC consiste d'abord à se saisir des discours scientifiques avant de les reformuler dans une version plus proche des gens du littoral et plus adaptée à leur propre territoire. Sur de telles bases, les animations proposées par l'association organisent la rencontre de la science et de l'émotion, de la raison et de la passion autour d'un objet commun. Chaque rendez-vous est ainsi l'occasion de faire jouer aux experts du quotidien, sortis des rangs des professionnels de la mer et du littoral, un rôle de passeur tout à fait original. Pêcheurs, conchyliculteurs, agriculteurs ou plaisanciers sont dépositaires d'un legs de pratiques et de savoirs essentiels. Parmi eux, les anciens présentent l'immense avantage d'ancrer leur expérience dans une temporalité - l'échelle d'une vie humaine - qui est à la fois celle des riverains qui les écoutent, celle des projections climatiques mais aussi celle des aménageurs qui doivent penser le littoral de demain face à ces changements. Leur expérience vécue, riche des mutations et des bouleversements dont ils ont été les témoins, délimite les champs du possible et de son acceptabilité pour demain. 


\section{REMERCIEMENTS}

Les auteurs remercient Jacques Boucard, Arnaud Dautricourt et Célia Levinet, membres du conseil d'administration de l'Université Populaire du Littoral Charentais, ainsi que Nathalie Giloy et Charly Point, stagiaires 2013 à l'UPLC pour leur aide à la rédaction de cet article.

\section{REFERENCES}

Breilh J-F., Bertin X., Chaumillon E., Giloy N., Sauzeau T. (2014) - How frequent is storm-induced flooding in the central part of the Bay of Biscay? Global and Planetary change. 122 161-175

Garnier E., Surville F. Dir. (2010) — La tempête Xynthia face à l'histoire. Submersions et tsunamis sur les littoraux français du Moyen Âge à nos jours Le Croît Vif. 2010 146-148

GARNIER E. (2010) - La crise Xynthia à l'aune de l'histoire. Enseignements et enjeux contemporains d'une histoire des submersions. Contribution aux missions d'enquête parlementaire et sénatoriale sur Xynthia par le groupe de recherche Submersions

Goeldner-Gianella L. (2013) - Dépoldériser en Europe occidentale. Pour une géographie et une gestion intégrées du littoral. Paris, Sorbonne. $340 \mathrm{p}$

Sauzeau T., ACERra M. (2012) - Zones construites, zones désertes sur le littoral atlantique français : les leçons du passé

Xynthia, regards de la géographie, de l'histoire et du droit, Norois, environnement, aménagement, société, Rennes. 222 p.123-143

Sauzeau T., Peret J. (2014) - Xynthia ou la mémoire réveillée. Villages charentais et vendéens face à la mer $\left(X V I I^{e}-X X I^{e}\right.$ siècles), La Crèche, Geste éditions. $289 \mathrm{p}$

SAuzeau T. (2014) — « Une défense active : l'usage de la mémoire des zones humides face au risque de submersion ». Zone s humides info. 82-83 p.5-6

SAUZEAu T. (2014) - L'histoire, les tempêtes et la prospective littorale face aux changements climatiques S'adapter à la mer L'homme, la mer et le littoral du Moyen Âge à nos jours, Enquêtes et documents, Nantes, CRHiA, PUR. 71-88 